\title{
OSTEONECROSE DOS MAXILARES ASSOCIADA AO USO DE BIFOSFONATOS: UMA REVISÃO DE LITERATURA
}

\section{OSTEONECROSIS OF THE JAWS ASSOCIATED WITH BISPHOSPHONATES: A LITERATURE REVIEW}

\author{
Luisa Machado Barin* \\ Fernanda Maia Pillusky* \\ Marcela Mozzaquatro Pasini** \\ Cristiane Cademartori Danesi"**
}

\begin{abstract}
RESUMO
Os Bifosfonatos (BFS) são fármacos antirreabsortivos frequentemente utilizados no tratamento de desordens ósseas e de neoplasias malignas metastáticas. Seu mecanismo de ação consiste na inibição da atividade osteoclástica e angiogênica do tecido ósseo. No entanto, o uso crônico desses medicamentos pode desenvolver a osteonecrose dos maxilares associada aos bifosfonatos (OMAB), principalmente em pacientes submetidos a traumas prévios. O presente trabalho objetiva revisar abordagens terapêuticas para esses pacientes, focando na prevenção e tratamento dessas patologias. A literatura tem apresentado propostas variadas para o tratamento da $\mathrm{OMAB}$, muitas vezes controverso. O tratamento dessas patologias tem se tornado desafiador, uma vez que os protocolos nem sempre são eficientes para controlar tal condição. Assim, previamente à terapêutica com BFS, os profissionais de saúde devem realizar todos os procedimentos necessários para adequação do meio bucal dos pacientes, bem como manter um rigoroso acompanhamento da sua condição bucal. Diante disso, é de fundamental importância a ação multidisciplinar entre médicos e cirurgiões-dentistas no manejo desses pacientes.

Descritores: Odontologia. Difosfonatos. Mandíbula. Maxila. Terapêutica.
\end{abstract}

\section{ABSTRACT}

Bisphosphonates (BPS) are antiresorptive agents routinely used in the management of bone diseases and metastatic malignancies neoplasm. Its mechanism of action inhibits the osteoclastic and angiogenic activity of bone tissue. However, its chronic use can raise Bisphosphonate-Related Osteonecrosis of the Jaws (BRONJ), mainly in patients submitted to previous trauma. This study aims to review therapeutic approaches of these patients, focusing on prevention and treatment of these pathologies. The literature has presented various proposals to treat the BRONJ, but sometimes it's controversial. The treatment of these pathologies has been challenging, once the protocols are not always effective to control this condition. Thus, previously of BFS therapeutic, the health professional should realize all the needed procedures to adequate the oral environment of these patients, as well as to maintain a rigorous attendance of your oral condition. It's essential the multidisciplinary approach between physician and dental surgeon to manage these patients.

Descriptors: Odontology. Diphosphonates. Mandible. Maxilla. Therapeutics.

\footnotetext{
* Doutoranda do Curso de Pós-graduação de Ciências Odontológicas com ênfase em Patologia Bucal pela UFSM, RS, Brasil. (luisambarin@hotmail.com) ** Mestre em Ciências Odontológicas com ênfase em Patologia Bucal pela UFSM, RS, Brasil. (fernandampillusky@hotmail.com, marcelam4p@gmail.com) *** Professora Doutora Adjunta do Departamento de Patologia da UFSM, RS, Brasil. (cristianedanesi@gmail.com)
} 


\section{N T R O D U Ç Ã O}

Os bifosfonatos (BFS) são drogas conhecidas desde a metade do século XIX, e sua primeira síntese farmacêutica ocorreu na Alemanha, em 1865. São análogos sintéticos não metabólicos dos pirofosfatos, compostos farmacológicos caracterizados pela alta afinidade ao tecido ósseo, pois possuem propriedades antiosteoclásticas e antiangiogênicas, que alteram o metabolismo ósseo. Esses medicamentos são prescritos para neoplasias malignas metastáticas e patologias de lise óssea ${ }^{1,2,3}$.

Estima-se que 3 milhões de pacientes com câncer no mundo tenham recebido tratamento com $\mathrm{BFS}^{4}$. O seu uso crônico pode suscitar a osteonecrose dos maxilares associada aos BFS (OMAB) como efeito colateral, ocorrendo em 1 a cada 100.000 pessoas por ano de exposição $0^{5,6}$, 7. Em 2003, essa patologia foi descrita pela primeira vez na literatura e, desde então, têm sido relatados inúmeros casos, devido à ampla indicação dos BFS e à escassez de conhecimento sobre tal patologia ${ }^{8}$. A OMAB pode se desenvolver espontaneamente ou em decorrências de traumas, e sua frequência e intensidade têm relação com o tipo de medicamento, a via de administração e a dosagem do fármaco ${ }^{9}$.

Como medidas preventivas, é essencial uma acurada anamnese, exame físico e radiográfico completo previamente ao início do uso de $\mathrm{BFS}^{10}$. O correto diagnóstico da OMAB desempenha um papel fundamental no desenvolvimento de estratégias terapêuticas para os pacientes portadores dessa condição. Uma vez instalada, a OMAB tem apresentado comportamento clínico preocupante e diversos tratamentos têm sido propostos, dentre eles emprego de soluções antimicrobianas, antibioticoterapia e procedimentos cirúrgicos mais agressivos, além de descontinuidade no uso de BFS, porém muitas vezes sem êxito ${ }^{11,12}$. A importância de uma equipe multidisciplinar, bem como de um rigoroso controle nas condições bucais e sistêmicas tornam-se essenciais para que se estabeleçam critérios para acompanhamento odontológico dos pacientes suscetíveis de complicações bucais decorrentes dessa terapia ${ }^{13,14}$.
O tratamento da OMAB tem se tornado desafiador, uma vez que ainda não existem formas totalmente eficientes de controle dessa patologia, pois suas estratégias de tratamento possuem prognóstico duvidoso. Contudo, parece haver predileção por tratamentos conservadores de combate da sintomatologia colateral ${ }^{15}$. Desse modo, o objetivo deste trabalho é elucidar sobre a abordagem de pacientes em terapia com BFS, focando as medidas de prevenção e estratégias de tratamento.

\section{REVISÃO DA LITERATURA}

Os BFS são fármacos sintéticos, análogos aos pirofosfatos endógenos, capazes de aderir à matriz óssea e provocar alterações no seu remodelamento ${ }^{16,17}$. O medicamento tem alta afinidade pela hidroxiapatita, principal componente do tecido ósseo, e atua inibindo a função osteoclástica e minimizando o turnoverósseo, através da estimulação de osteoblastos e inibição ou apoptose de osteoclastos ${ }^{16}$. Esses fármacos afetam o turnover ósseo a nível tecidual (inibem a reabsorção óssea); a nível celular (inibem a função osteoclástica: recrutamento, diminuição do tempo de vida e inibição da atividade na superfície óssea) e a nível molecular (por inibição de fatores de crescimento endoteliais e ligação ao receptor da superfície da célula ou uma enzima intracelular) ${ }^{14,18,19,20 .}$

Os BFS estão entre as noventa drogas mais prescritas no mundo ${ }^{21}$, pois têm proporcionado aos pacientes uma melhora na qualidade de vida em relação a dor e eventos indesejados de origem óssea. A prescrição desse fármaco é realizada com o intuito de prevenir e tratar patologias ósseas, como osteoporose, osteogênese imperfeita, displasia fibrosa, doença de Paget, mieloma múltiplo e neoplasias malignas metastáticas, principalmente decorrentes de câncer de mama e de próstata $^{17,22}$.

As propriedades antirreabsortivas dos BFS potencializam-se entre as diferentes gerações da droga. A primeira geração inclui o etidronato e clodronato, a segunda compreende os aminobifosfonatos, como o alendronato e o pamidronato, e a terceira geração é representada pelo risedronato e o zoledronato. Estes ainda são
BARIN LM

PILLUSKY FM

PASINI MM

DANESI CC

OSTEONECROSE

DOS MAXILARES

ASSOCIADA

AO USO DE

BIFOSFONATOS:

UMA REVISÃO DE

LITERATURA

127

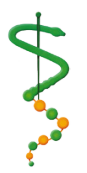

REV, ODONTOL.

UNIV. CID. SÃO

PAULO

$2016 ; 28(2):$

$126-34$, MAI - AGO 
BARIN LM

PILLUSKY FM

PASINI MM :

DANESI CC :

OSTEONECROSE

DOS MAXILARES

ASSOCIADA

$A O$ USO DE

BIFOSFONATOS:

UMA REVISÃO DE LITERATURA

$128 \cdots$

REV. ODONTOL. UNIV. CID, SÃO

PAULO

$2016 ; 28(2):$ $126-34$, MA I - AGO divididos em dois subgrupos, de acordo com a ausência (etidronato) e a presença (alendronato, zoledronato, risedronato) de nitrogênio nas suas cadeias laterais. A associação entre pamidronato e zoledronato tem demonstrado maior desenvolvimento da $\mathrm{OMAB}^{17,22,23}$.

Apesar do efeito benéfico da droga, a literatura aponta que há um efeito colateral sugestivo do uso crônico dos BFS, denominado OMAB. A osteonecrose apresenta comportamento indolente e de difícil controle, definida pela exposição óssea espontânea ou por feridas não cicatrizantes, que podem ou não estar associadas a infecção, fístula ou fratura. Tal manifestação associada aos BFS passou a estar localizada quase exclusivamente nos maxilares, sendo então denominada Osteonecrose dos Maxilares associada ao uso de BFS $(\mathrm{OMAB})^{7}$. Em 2007, a American Association of Oral and Maxillofacial Surgeons $^{11}$ estabeleceu parâmetros para a identificação da $\mathrm{OAB}$, composto pela existência simultânea dos seguintes fatores: a) uso de bifosfonatos atual ou prévio; b) presença de necrose óssea na região maxilofacial que persista por mais de 8 semanas e c) inexistência de história de radioterapia local.

Algumas características epidemiológicas da OMAB podem ser observadas. Sua incidência ocorre duas vezes mais na mandíbula do que na maxila, principalmente na superfície lingual da região posterior e em áreas de fina mucosa sobre proeminências ósseas. Assim como ambos os ossos podem ser afetados simultaneamen$\mathrm{te}^{18,24}$. As mulheres são frequentemente mais afetadas que os homens, visto que há maior incidência do câncer de mama e maior acometimento de patologias de lise óssea, especialmente a osteoporose ${ }^{17}$. A faixa etária de maior acometimento é a $7^{a}$ década de vida.

O tipo de medicamento, a via de administração, a dosagem e a duração do tratamento com BFS desempenham um papel fundamental no surgimento da OMAB (Melea). A via endovenosa (pamidronato e o zoledronato) está relacionada ao tratamento de pacientes oncológicos e a via oral (alendronato, risendronato e ibandronato) associada ao tratamento de doenças que acarretam a lise óssea 22,25 . A alta seletividade e a deposição óssea são obtidas pelos medicamentos endovenosos, sendo absorvidos aproximadamente $50 \%$ da dose, a qual permanece ligada ao osso por longos períodos, atingindo até 10 anos $^{22,}$ 26. Nos casos de administração via oral, o fármaco enfrenta a barreira estomacal e apenas $1 \%$ é absorvido e incorporado ao osso. Ainda, os pacientes que recebem BFS endovenoso e são submetidos à cirurgia dentoalveolar possuem no mínimo sete vezes mais chances de desenvolver osteonecrose do que pacientes que não são submetidos a tal procedimento ${ }^{11}$.

A OMAB pode se desenvolver em decorrência de traumas na região bucal ou espontaneamente. A predisposição para a necrose óssea nos maxilares está relacionada a microtraumas constantes, oriundos dos movimentos mandibulares e da falta da adequada remodelação óssea, decorrente da inibição osteoclástica e da insuficiência vascular, tornando-se incapazes de fazer o osso responder adequadamente às exigências metabólicas ${ }^{9,27,28}$. A presença da microbiota oral pode desencadear infecções secundárias e dificultar o processo cicatricial. Coelho et al. ${ }^{8}$ (2010) sugerem alguns fatores de risco para $\mathrm{O}$ desenvolvimento da OMAB relacionados às condições bucais como extrações dentárias, cirurgias orais com manipulação óssea, próteses mal adaptadas, doença periodontal prévia, corroborando para a gênese OMAB. Ainda, o elevado consumo de álcool e fumo, uso de glicocorticoides e comorbidades sistêmicas preexistentes podem contribuir para o desenvolvimento dessa lesão $\mathrm{O}^{23,29,30 .}$.

Alguns pacientes permanecem assintomáticos por várias semanas ou meses, apresentando como alteração apenas a presença de osso exposto na boca. As lesões passam a ser sintomáticas quando infeccionadas secundariamente, ou por trauma local proveniente de espículas do osso exposto ${ }^{31}$. Se a infecção tornar-se aguda, pacientes podem ainda queixar-se de dor exacerbada até perda sensorial ${ }^{32} \mathrm{ou}$ parestesia devido à proximidade com terminações nervosas ${ }^{33}$.

Clinicamente, a osteonecrose pode apresentar um amplo espectro de mani- 
festações. O diagnóstico diferencial é de extrema importância para o manejo da $\mathrm{OMAB}^{34}$. Nos estágios iniciais, a OMAB pode mimetizar as características clínicas e radiográficas de diversas patologias orais, como periodontite, mucosite, disfunção temporomandibular, sinusite, entre outros, apresentando mobilidade dentária, perda óssea e supuração. A partir disso, é necessário um correto diagnóstico pelo cirurgião-dentista, uma vez que são patologias distintas que requerem atenção e manejos terapêuticos diferentes ${ }^{35}$. A melhor compreensão da atuação dos BFS na patogenia da OMAB é essencial para a adoção de um planejamento preventivo ${ }^{36}$.

Previamente ao início do tratamento com BFS, deve-se ter em mente alguns cuidados que serão cruciais para prevenir o desenvolvimento da OMAB. É imprescindível que o paciente procure por atendimento odontológico para avaliação das suas condições bucais, incluindo-se exames clínico e radiográfico cuidadosos e completos para verificar a necessidade de adequação do meio bucal ${ }^{37,38}$. Presença de cárie e doença periodontal, fatores retentivos de placa, raízes residuais, focos de infecção, cirurgias periodontais, dentes que necessitam ser extraídos e de tratamento de canal são fatores a serem considerados durante o exame clínico do paciente $^{8,39}$. Se as condições sistêmicas do paciente o permitirem, é recomendável que o início do tratamento com BFS seja adiado por pelo menos um mês para adequada cicatrização óssea ou até que se reúnam todas as condições para uma boa saúde bucal. Entretanto, essa é uma medida que deve sempre ser tomada em conjunto com a equipe médica do pacien$\mathrm{te}^{23,37}$.

Após a prescrição do uso de BFS para o paciente, este deve passar por monitoramento semestral da sua saúde bucal, medidas profiláticas e instruções de higiene constantes, com o intuito de minimizar o surgimento da $\mathrm{OMAB}^{32,40}$. Geralmente, não é necessário interromper o tratamento para a realização de algum procedimento odontológico, porém devem-se evitar cirurgias traumáticas e lesões de fricção crônica. O paciente que faz uso de BFS deve sempre ser alertado sobre os possíveis ris- cos diante da realização dos procedimentos, bem como sobre os sinais e sintomas decorrentes do surgimento da OMAB, devendo sempre procurar seu médico e/ou cirurgião-dentista ${ }^{41,42,43}$.

Uma vez estabelecido o diagnóstico de OMAB, a padronização da melhor conduta terapêutica tem se tornado um desafio. Na tentativa de nortear essas modalidades, a OMAB foi classificada em 3 estágios: estágio clínico 1 , representado por pacientes com osso necrótico exposto assintomático; estágio 2, osso necrótico exposto associado à dor e infecção; e estágio 3, osso necrótico exposto associado à dor, infecção, fratura patológica, fístula extraoral ou osteólise estendendo-se ao bordo inferior ${ }^{44}$. A literatura tem proposto terapêuticas baseadas nesses estágios: bochechos de clorexidina $0,12 \%$ (estágio 1); bochechos e antibioticoterapia (estágio 2) e tratamentos mais agressivos como ressecção associada à antibioticoterapia (estágio 3) (23). Essas terapias são variadas e integram o uso de antibióticos, debridamento local, irrigação local com soluções antimicrobianas, ressecções cirúrgicas, sequestrectomia, oxigenação hiperbárica, laserterapia, uso de plasma rico em plaquetas, ozonioterapia e baixas doses de paratormônio ${ }^{9,11,44}$. Entretanto, os estudos afirmam predileção por tratamentos não invasivos, apenas para controle da sintomatologia, da infecção da progressão da lesão ${ }^{10,44}$. Muitas vezes, a cura completa não é obtida e os pacientes podem conviver com algum grau de exposição óssea por anos ${ }^{28}$.

A OMAB, quando instalada, frequentemente persiste por um longo período, gerando extensas áreas de exposição óssea e deiscências progressivas de difícil tratamento ${ }^{32}$. Os diferentes tratamentos propostos têm apresentado resultados controversos e com pouco embasamento científico ${ }^{42}$. Atualmente, não existe um tratamento único que seja eficaz e a combinação de ciclos de antibioticoterapia com tratamentos cirúrgicos simples tem oferecido a melhor hipótese de cura ${ }^{45}$. Entretanto, alguns autores conseguiram controle e limitação da progressão das lesões com antibioticoterapia intermitente, uso de colutórios à base de clorexidina e de-
BARIN LM

PILLUSKY FM

PASINI MM

DANESI CC

OSTEONECROSE

DOS MAXILARES

ASSOCIADA

AO USO DE

BIFOSFONATOS:

UMA REVISÃO DE

LITERATURA

129

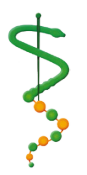

REV, ODONTOL.

UNIV, CID , SÃO

PAULO

$2016 ; 28(2)$

$126-34$, MA I - AGO 
BARIN LM

PILLUSKY FM

PASINI MM :

DANESI CC

OSTEONECROSE

DOS MAXILARES

ASSOCIADA

AO USO DE

BIFOSFONATOS:

UMA REVISÃO DE

LITERATURA

$130 \ldots$

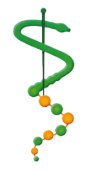

REV. ODONTOL. UNIV, CID, SÃO

PAULO

$2016 ; 28(2):$ $126-34$, MA I - AGO bridamento periódico do osso sequestrado com irrigação da ferida ${ }^{35,43}$. Freiberger et al. ${ }^{46}$ (2007) sugerem que a oxigenação hiperbárica pode ser efetiva na redução do tamanho das lesões, porém esse efeito tem sido maior quando associado à descontinuidade medicamentosa dos BFS.

Mediante a ausência de unanimidade no que tange o estabelecimento de protocolos de tratamentos seguros e eficazes, a terapêutica é cirúrgica quando há sintomatologia dolorosa, bem como presença de fraturas patológicas, osso necrosado, como fonte de infecção, ou quando os tratamentos conservadores se mostrarem ineficazes $^{10}$. O tratamento cirúrgico é frequentemente utilizado, porém deve ser evitado para não exacerbar os quadros de exposição óssea e facilitar a entrada dos microrganismos ${ }^{25,47}$. Para pacientes que não responderam bem ao tratamento medicamentoso, a ressecção cirúrgica tem sido considerada como um método paliativo de resolução da doença ${ }^{23}$.

Controversamente, Marx et al. ${ }^{48}$ (2003) relataram uma série de 36 casos em que os pacientes estavam em tratamento com BFS e tiveram exposições ósseas dolorosas em maxila e/ou mandíbula, que não responderam ao tratamento cirúrgico. $\mathrm{Na}$ maioria dos casos, os autores conseguiram controle e limitação da progressão das lesões com antibioticoterapia intermitente, uso de colutórios à base de clorexidina e debridamento periódico do osso sequestrado com irrigação da ferida. Magopoulos et al. ${ }^{49}$ (2007) relatam que a associação do debridamento cirúrgico para obtenção de margens limpas e sangrantes com antibioticoterapia ainda permanece a melhor opção. Todavia, Scarpa et al. ${ }^{23}$ (2010) não recomendam o debridamento cirúrgico, uma vez que a falta de suprimento sanguíneo não acarreta adequada cicatrização óssea e exposição óssea decorrente desse procedimento pode piorar os sintomas e aumentar o risco de fratura nos maxilares.

A descontinuidade da terapêutica com BFS é um aspecto de extrema relevância. Dada a longa meia-vida desses fármacos no organismo e sua ativa ligação com o osso, sua cessação não modifica a história natural da doença e, consequentemente, pode não produzir efeitos imediatos na melhoria do quadro clínico ${ }^{12,50}$. Além disso, na maioria dos estudos com pacientes oncológicos, o uso continuado de BFS é essencial para a sobrevida do paciente ${ }^{11}$, 32. A descontinuação do tratamento BFS antes de procedimento cirúrgico dentário não parece evitar a OMAB. Em contrapartida, Ferreira Junior et al. ${ }^{42}$ (2007) ressaltam que pacientes que utilizam BFS associados a glicocorticoides devem suspender seu uso por 3 meses antes do procedimento cirúrgico, só devendo retomar após completa cicatrização óssea. Atualmente, o que é aceito é que a descontinuidade dos BFS deve ser ponderada caso a caso, com discussão dos riscos e benefícios aí inerentes e sempre deve ser realizado pela equipe médica que os prescreveu ao paciente ${ }^{42}$.

Até que maiores informações estejam disponíveis, seria prudente considerar todos os pacientes que tomam BFS como portadores de algum risco para osteonecrose associada a essa substância, com o reconhecimento de que a magnitude do risco provavelmente varia, dependendo do consumo individual da droga, fatores do paciente (interação medicamentosa, doenças prévias, fatores comportamentais) e histórico de tratamento odontológico. Ainda na ocasião do procedimento cirúrgico, é fundamental um exame clínico detalhado, uma anamnese que valorize perguntas sobre o uso de bifosfonato e, quando necessário, uma cobertura antibiótica anterior à cirurgia.

\section{CONSIDERAÇÕES FINAIS}

O uso de BFS por longos períodos é sugestivo para o aparecimento de necrose nos ossos gnáticos e o manejo dos pacientes portadores dessa patologia tem gerado amplas discussões. Uma vez estabelecida, a OMAB dificilmente será controlada pela resposta de defesa do hospedeiro, visto que os BFS alteram o metabolismo sistêmico do tecido ósseo.

Por ser uma condição relativamente recente descrita na literatura, a inexistência de um protocolo efetivo torna o tratamento da OMAB desafiador. As tentativas de realizar o debridamento completo e recobrir o osso exposto têm sido, quase sempre, 
contraproducentes, levando à maior exposição do osso necrótico e agravamento dos sinais e sintomas, desenvolvendo um maior risco para fraturas patológicas. O profissional deve ficar atento aos fatores de risco que podem influenciar na osteonecrose, prestando atenção à idade do paciente, tempo de uso dos bifosfonatos, modo de utilização dessas drogas (injetáveis ou não) e a existência de traumas. $\mathrm{O}$ melhor a se fazer é evitar procedimentos invasivos, já que, quanto mais extensa a cirurgia, maiores os riscos.

A melhor conduta ainda é a preven- ção e, desse modo, a importância de uma equipe multidisciplinar, bem como de um rigoroso controle nas condições bucais e sistêmicas são peças chaves para o manejo desses pacientes. Quando instalada a OMAB, o controle de infecções, a diminuição na progressão da lesão e o alívio da dor podem resultar no bem-estar geral e aumento da qualidade de vida dos mesmos. É interessante que estudos sobre OMAB persistam, para que protocolos de tratamento sejam estabelecidos e indicados de forma segura e eficaz.

\section{REFERÊNCIAS}

1. Fleisch H. Development of bisphosphonates. Breast Cancer Res 2002 4(1):30-4.

2. Ott SM. Long-term safety of bisphosphonates. J Clin Endocrinol Metab 2005 Mar;90(3):1897-9.

3. Gegler A, Cherubini K, Figueiredo MAZ, Yurgel LS, Azambuja AA. Bisfosfonatos e osteonecrose maxilar : revisão da literatura e relato de dois casos. Rev Bras Cancerol 2006 52(1):25-31.

4. Sahni M, Guenther HL, Fleisch H, Collin P, Martin TJ. Bisphosphonates act on rat bone resorption through the mediation of osteoblasts. J Clin Invest 1993 May;91(5):2004-11.

5. Leite AF, Santos Ogata F, Melo NS, Figueiredo PTS. Imaging findings of bisphosphonate-related osteonecrosis of the jaws: a critical review of the quantitative studies. Int J Dent 2014 $1-11$.

6. Khosla S, Burr D, Cauley J, Dempster DW, Ebeling PR, Felsenberg D, et al. Bisphosphonate-associated osteonecrosis of the jaw: report of a task force of the American Society for Bone and Mineral Research. J Bone Miner Res 2007 Oct;22(10):1479-91.
7. Longo F, Guida A, Aversa C, Pavone E, Di Costanzo G, Ramaglia L, et al. Platelet rich plasma in the treatment of bisphosphonate-related osteonecrosis of the jaw: personal experience and review of the literature. Int J Dent 2014 2014(1):1-7.

8. Coelho AI, Gomes PS, Fernandes $\mathrm{MH}$. Osteonecrose dos maxilares associada ao uso de bifosfonatos. parte II: inhas de orientação na consulta de medicina dentária. Rev Port Estomatol Med Dentária Cir Maxilofac 2010 2010/07/01;51(3):185-91.

9. Ruggiero SL, Mehrotra B, Rosenberg TJ, Engroff SL. Osteonecrosis of the jaws associated with the use of bisphosphonates: a review of 63 cases. J Oral Maxillofac Surg 2004 May;62(5):527-34.

10. Pereira FA, Pereira JC, Pereira CAA, Carvalho RWF, Antunes AA. Osteonecrose dos maxilares associada a bisfosfonatos. Rev Bras Cir Cabeça Pescoço 2009 out/nov/dez;38(4):283-6.

11. American Association of Oral and Maxillofacial Surgeons position paper on bisphosphonate-related osteonecrosis of the jaws. J Oral Maxillofac Surg 2007 Mar;65(3):369-76.
BARIN LM PILLUSKY FM PASINI MM DANESI CC

OSTEONECROSE DOS MAXILARES ASSOCIADA AO USO DE BIFOSFONATOS: UMA REVISÃO DE LITERATURA

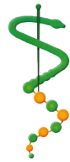

REV, ODONTOL. UNIV. CID. São PAULO

2016; 28(2): $126-34$, MA I - AGO 
BARIN LM

PILLUSKY FM

PASINI MM :

DANESI CC

OSTEONECROSE

DOS MAXILARES

AS SOCIADA

AO USO DE

BIFOSFONATOS:

UMA REVISÃO DE LITERATURA

132

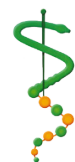

REV. ODONTOL.

UNIV, CID, SÃO:

PAULO

2016; 28(2):

$126-34$, MAI - AGO
12. Marx RE, Cillo JE, Jr., Ulloa JJ. Oral bisphosphonate-induced osteonecrosis: risk factors, prediction of risk using serum CTX testing, prevention, and treatment. J Oral Maxillofac Surg 2007 Dec;65(12):2397-410.

13. Tanvetyanon T, Stiff PJ. Management of the adverse effects associated with intravenous bisphosphonates. Ann Oncol 2006 Jun; 17(6):897-907.

14. Souza LN, Antunes Souza ACR, Arruda Mari VF, Nogueira Borges AP, Alvarenga RL. Osteonecrose dos maxilares associada ao uso de bisfosfonatos: revisão da literatura e apresentação de um caso clínico. Rev Port Estomatol Med Dentária Cir Maxilofac 2009 50(4):229-36.

15. Gebara SN, Moubayed H. Risk of osteonecrosis of the jaw in cancer patients taking bisphosphonates. Am J Health Syst Pharm 2009 Sep 1;66(17):1541-7.

16. Franco S, Miccoli S, Limongelli L, Tempesta A, Favia G, Maiorano E. New dimensional staging of bisphosphonate-related osteonecrosis of the jaw allowing a guided surgical treatment protocol: long-term follow-up of 266 lesions in neoplastic and osteoporotic patients from the university of bari. Int J Dent 2014 2014(1):1-10.

17. Sarkarat F, Kalantar Motamedi MH, Jahanbani J, Sepehri D, Kahali R, Nematollahi Z. Platelet-rich plasma in treatment of zoledronic acid-Induced bisphosphonate-related osteonecrosis of the jaws. Trauma Mon 2014 Apr;19(2):e17196.

18. Nunes V, Lopes B, Lordani RXF, Alves J, Rocha R, Machado W, et al. Uso de bifosfonatos em pacientes com câncer e sua associação com osteonecrose dos ossos maxilares: uma revisão de literatura. Rev Periodontia 2010 set;20(3):20-7.

19. Sousa FRN, Jardim Junior EG. Osteonecrose associada com o uso dos bifosfonatos. Pesq Bras Odontoped Clin Integr 2008 set/dez;8(3):375-80.
20. Pastor-Zuazaga D, Garatea-Crelgo J, Martino-Gorbea R, Etayo-Perez A, Sebastian-Lopez C. Osteonecrosis of the jaws and bisphosphonates. Report of three cases. Med Oral Patol Oral Cir Bucal 2006 Jan;11(1):E76-9.

21. Harper RP, Fung E. Resolution of bisphosphonate-associated osteonecrosis of the mandible: possible application for intermittent low-dose parathyroid hormone [rhPTH(1-34)]. J Oral Maxillofac Surg 2007 Mar;65(3):57380.

22. Sigua-Rodriguez EA, da Costa Ribeiro $\mathrm{R}$, de Brito AC, Alvarez-Pinzon N, Albergaria-Barbosa JR. Bisphosphonaterelated osteonecrosis of the jaw: a review of the literature. Int J Dent 2014 2014(1):1-5.

23. Scarpa LC, Leite LCM, Lacerda JCT, Arantes DCB. Osteonecrose nos ossos da maxila e mandíbula associada ao uso do bifosfonato de sódio. Rev Bras Pesqui Saude 2010 12(1):86-92.

24. Marx RE, Sawatari Y, Fortin M, Broumand V. Bisphosphonate-induced exposed bone (osteonecrosis/osteopetrosis) of the jaws: risk factors, recognition, prevention, and treatment. J Oral Maxillofac Surg 2005 Nov;63(11):156775.

25. Martins MAT, Giglio AD, Martins MD, Pavesi VCS, Lascala CA. Osteonecrose dos maxilares associada ao uso de bisfosfonatos: importante complicação do tratamento oncológico. Rev Bras Hematol Hemoter 2009 fev;31(1):416.

26. Walter C, Grotz KA, Kunkel M, Al-Nawas $B$. Prevalence of bisphosphonate associated osteonecrosis of the jaw within the field of osteonecrosis. Support Care Cancer 2007 Feb;15(2):197202.

27. Whyte MP, Wenkert D, Clements KL, McAlister WH, Mumm S. Bisphosphonate-induced osteopetrosis. N Engl J Med 2003 Jul 31;349(5):457-63. 
28. Hoefert S, Eufinger H. Sunitinib may raise the risk of bisphosphonate-related osteonecrosis of the jaw: presentation of three cases. Oral Surg Oral Med Oral Pathol Oral Radiol Endod 2010 Oct;110(4):463-9.

29. Ficarra G, Beninati F. Bisphosphonaterelated Osteonecrosis of the Jaws: An Update on Clinical, Pathological and Management Aspects. Head Neck Pathol 2007 1(2):132-40.

30. Passeri LA, Bértolo MB, Abuabara A. Osteonecrose dos maxilares associada ao uso de bisfosfonatos. Rev Bras Reumatol 2011 ago;51(4):404-7.

31. Gutta R, Louis PJ. Bisphosphonates and osteonecrosis of the jaws: science and rationale. Oral Surg Oral Med Oral Pathol Oral Radiol Endod 2007 Aug;104(2):186-93.

32. Migliorati CA, Casiglia J, Epstein J, Jacobsen PL, Siegel MA, Woo SB. Managing the care of patients with bisphosphonate-associated osteonecrosis: an American Academy of Oral Medicine position paper. J Am Dent Assoc 2005 Dec;136(12):1658-68.

33. Melo MD, Obeid G. Osteonecrosis of the jaws in patients with a history of receiving bisphosphonate therapy: strategies for prevention and early recognition. J Am Dent Assoc 2005 Dec;136(12):1675-81.

34. Carvalho A, Mendes RA, Carvalho DCJ. Osteonecrose da mandíbula associada a bifosfonatos intravenosos em doentes oncológicos. Acta Med Port 2008 21(5):505-10.

35. Silverman SL, Landesberg R. Osteonecrosis of the jaw and the role of bisphosphonates: a critical review. Am J Med 2009 Feb;122(2 Suppl):S33-45.

36. Campisi G, Fedele S, Fusco V, Pizzo G, Di Fede O, Bedogni A. Epidemiology, clinical manifestations, risk reduction and treatment strategies of jaw osteonecrosis in cancer patients exposed to antiresorptive agents. Future Oncol 2014 Feb;10(2):257-75.
37. Silva AS, Conceição TS, Veloso KMM, Cartágenes MSS. Análise de prontuários de pacientes com câncer de mama em tratamento com bisfosfonatos: fator de risco para manifestações orais e osteonecrose induzida. Rev Soc Bras Clín Méd 2013 jul-set;11(3):242-5.

38. Khan AA, Sandor GK, Dore E, Morrison AD, Alsahli M, Amin F, et al. Canadian consensus practice guidelines for bisphosphonate associated osteonecrosis of the jaw. J Rheumatol 2008 Jul;35(7):1391-7.

39. Assael LA. New foundations in understanding osteonecrosis of the jaws. J Oral Maxillofac Surg 2004 Feb;62(2):125-6.

40. Migliorati CA, Armonis BN, NicolatouGalitis O. Oral osteonecrosis associated with the use of ibandronate: report of a case and clinical implications. Oral Surg Oral Med Oral Pathol Oral Radiol Endod 2008 Jul;106(1):e18-21.

41. Santos PSS, Gambirazi LM, Felix VB, Magalhães MHCG. Osteonecrose maxilar em pacientes portadores de doenças neoplásicas sob uso de bisfosfonatos. Rev Bras Hematol Hemoter 2008 dez;30(6):501-4.

42. Ferreira Junior CD, Casado PL, Barboza ESP. Osteonecrose associada aos bifosfonatos na odontologia. Rev Periodontia 2007 dez; 17(24-30):4.

43. Bamias A, Kastritis E, Bamia C, Moulopoulos LA, Melakopoulos I, Bozas G, et al. Osteonecrosis of the jaw in cancer after treatment with bisphosphonates: incidence and risk factors. J Clin Oncol 2005 Dec 1;23(34):8580-7.

44. Ruggiero SL, Fantasia J, Carlson E. Bisphosphonate-related osteonecrosis of the jaw: background and guidelines for diagnosis, staging and management. Oral Surg Oral Med Oral Pathol Oral Radiol Endod 2006 Oct;102(4):433-41.
BARIN LM PILLUSKY FM

PASINI MM

DANESI CC

OSTEONECROSE

DOS MAXILARES

ASSOCIADA

AO USO DE

BIFOSFONATOS:

UMA REVISÃO DE

LITERATURA

- 133

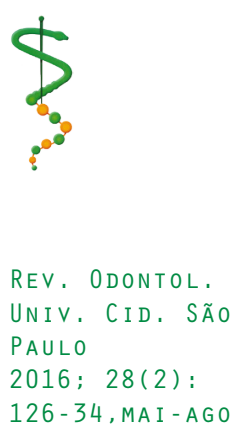


BARIN LM

PILLUSKY FM

PASINI MM

DANESI CC

OSTEONECROSE

DOS MAXILARES

ASSOCIADA

AO USO DE

BIFOSFONATOS:

UMA REVISÃO DE

LITERATURA

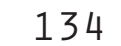

45. Lopes I, Zenha H, Costa H, Barroso J. Osteonecrose da mandíbula associada ao uso de bifosfonatos: uma patologia secundária grave. Arq Med 2009 23(5):181-5.

46. Freiberger JJ, Padilla-Burgos R, Chhoeu $\mathrm{AH}$, Kraft KH, Boneta O, Moon RE, et al. Hyperbaric oxygen treatment and bisphosphonate-induced osteonecrosis of the jaw: a case series. J Oral Maxillofac Surg 2007 Jul;65(7):13217.

47. Ruggiero SL, Drew SJ. Osteonecrosis of the jaws and bisphosphonate therapy. J Dent Res 2007 Nov;86(11):1013-21.

48. Marx RE. Pamidronate (Aredia) and zoledronate (Zometa) induced avascular necrosis of the jaws: a growing epidemic. J Oral Maxillofac Surg 2003 Sep;61(9):1115-7.
49. Magopoulos C, Karakinaris G, Telioudis Z, Vahtsevanos K, Dimitrakopoulos I, Antoniadis K, et al. Osteonecrosis of the jaws due to bisphosphonate use. A review of 60 cases and treatment proposals. Am J Otolaryngol 2007 May-Jun;28(3):158-63.

50. Gomez-Moreno G, Arribas-Fernandez MC, Fernandez-Guerrero M, Boquete-Castro A, Aguilar-Salvatierra A, Guardia J, et al. Bisphosphonate-associated osteonecrosis of the jaw 2 years after teeth extractions: a case report solved with non-invasive treatment. Eur Rev Med Pharmacol Sci 2014 18(9):13917.

Recebido em 07/06/2016

Aceito em 08/08/2016
REV. ODONTOL. UNIV, CID, SÃO PAULO $2016 ; 28(2):$ $126-34$, MA I - AGO 